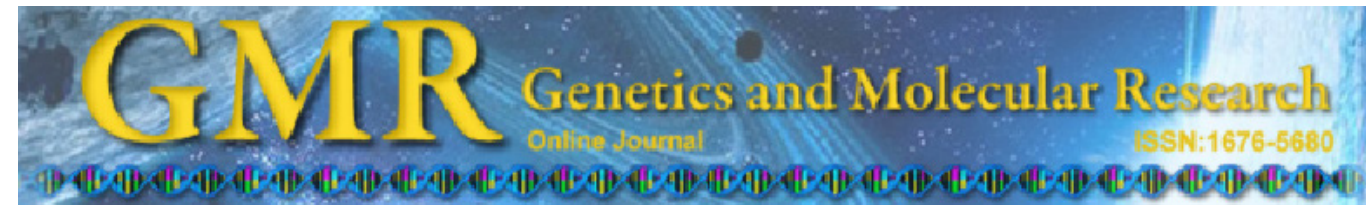

\title{
Intracellular localization and association of MHC class I with porcine invariant chain
}

\author{
F.Z. $X u^{1}$, S.G. $W u^{2}$ and W.Y. Yu ${ }^{1}$ \\ ${ }^{1}$ Anhui Agricultural University, Hefei, China \\ ${ }^{2}$ Anhui AnFengTang Animal Medicine Industry Co., Ltd., Anhui, Hefei, China \\ Corresponding author: F.Z. Xu \\ E-mail: xufazhi1103@sina.com
}

Genet. Mol. Res. 12 (1): 693-701 (2013)

Received May 17, 2012

Accepted August 20, 2012

Published March 11, 2013

DOI http://dx.doi.org/10.4238/2013.March.11.17

\begin{abstract}
The objective was to investigate the intracellular localization and association of pig major histocompatibility complex (MHC) class I subunits with invariant chain (Ii). Pig MHC class I subunit cDNAs were cloned by RT-PCR and eukaryotic expression plasmids of $\alpha$ and $\beta 2 \mathrm{~m}$ were constructed with fusions to red or enhanced green fluorescent protein (pDsRed2-N1- $\alpha$, pEGFP-N1- $\alpha$, pDsRed2-N1$\beta 2 \mathrm{~m}$, and $\mathrm{pEGFP}-\mathrm{N} 1-\beta 2 \mathrm{~m})$. A pig Ii mutant with a deleted CLIP region ( $\triangle$ CLIP-Ii) was constructed by overlap extension PCR. Wild-type Ii and mutant Ii were cloned into pEGFP-C1 (pEGFP-C1-Ii, pEGFP-C1$\triangle \mathrm{CLIP}-\mathrm{Ii})$. The recombinant plasmids of MHC I subunits and $\mathrm{pEGFP-}$ C1-Ii (pEGFP-C1- $\triangle$ CLIP-Ii) were transiently cotransfected into COS-7 cells with Lipofectamine 2000. Immunofluorescence microscopy was performed to detect expression and intracellular localization of Ii and MHC I subunits, and immunoprecipitation was used to analyze their association. Our results indicated that pig Ii associates with integrated MHC I subunits to form oligomers, but cannot associate with single MHC I subunits. Furthermore, deletion of the Ii CLIP sequence blocks association with integrated MHC I subunits. Thus, pig Ii cannot associate with a single MHC I molecule, the $\alpha$ or $\beta 2 \mathrm{~m}$ chain, but Ii and the integrated MHC I molecule can form complexes that colocalize in
\end{abstract}


the endomembranes of COS-7 cells. The Ii of CLIP plays a key role in assembly of Ii and MHC I.

Key words: Pig invariant chain; Intracellular localization; MHC I molecule

\section{INTRODUCTION}

Major histocompatibility complex (MHC) class I molecules bind endogenous peptide fragments and display them at the cell surface where they report the presence of antigen to CD8 ${ }^{+}$T cells (Roche and Cresswell, 1990; Teyton et al., 1990; Van Bleek and Nathenson, 1990). Exogenously derived peptides are presented to $C D 4^{+} \mathrm{T}$ lymphocytes in association with MHC class II (Odorizzi et al., 1994). The synthesis of MHC I and MHC II occurs at the endoplasmic reticulum, and they are sub-selected in the Golgi. MHC I is transported to the cell surface through the membrane bubble, while MHC II reaches the lysosomes (Teyton et al., 1990; Cresswell, 1992; Donaldson and Williams, 2009). These different transport routes of MHC I and MHC II may be caused by the invariant chain (Ii). Ii is a transmembrane glycoprotein, expressed primarily in antigen-presenting cells, which plays an important role in the assembly of the MHC class II-peptide complex. Within the endocytic compartment, Ii is proteolytically degraded to a short peptide called class II-associated invariant chain peptide (CLIP), which is exchanged for peptide (Peters et al., 1991; Romagnoli and Germain, 1994; Tulp et al., 1994).

In addition to binding to MHC class II molecules, Ii also associates with MHC class I molecules, although there has been little study of its effects on the MHC I molecule (Reber et al., 2002; Powis, 2006). Complexes of Ii and MHC class I molecules accumulate in the cis-Golgi and endosomes (Sugita and Brenner, 1995; Vigna et al., 1996). The interaction appears to require fully folded MHC class I. Open conformations of MHC class I or free heavy chains and $b_{2}$-microglobulin do not associate efficiently with Ii (Vigna et al., 1996; Reber et al., 2002). The Ii-MHC class I interaction also influences cell surface expression of MHC class I (Sugita and Brenner, 1995). Powis (2006) reported that the CLIP region is directly involved in the association of Ii with MHC class I molecules, and that this can result in the inclusion of Ii in the MHC class I peptide-loading complex. Recent research showed that Ii associated with MHC class I in the endoplasmic reticulum of DCs and mediated trafficking of MHC class I to endolysosomal compartments for loading with exogenous peptides (Basha et al., 2012).

Research on the pig Ii and MHC I has not been reported. It is unknown if pig Ii can associate with MHC I $\alpha$ or $\beta$ chain and whether its CLIP plays a key role in the assembly of Ii and MHC I. Therefore, we constructed eukaryotic expression plasmids of the pig MHC class I antigen presentation subunit fused with red (RFP) or enhanced green fluorescent protein (GFP). The recombinant plasmids were transiently transfected into COS-7 cells. Immunofluorescence microscopy was carried out to detect the expression and intracellular localization of Ii and MHC I subunits, and immunoprecipitation was used to analyze their association.

\section{MATERIAL AND METHODS}

\section{RNA extraction}

Total RNA was extracted from pig spleen lymphocytes using the Trizol reagent (In- 
vitrogen, Carlsbad, CA, USA) as previously described (Zhong et al., 2004). Briefly, samples were homogenized in $1 \mathrm{~mL}$ Trizol reagent and $0.2 \mathrm{~mL}$ chloroform was added to each sample; all samples were centrifuged at $12,000 \mathrm{~g}$ for $15 \mathrm{~min}$ at $4^{\circ} \mathrm{C}$ to separate the mixture. RNA in the aqueous phase was precipitated with $0.5 \mathrm{~mL}$ isopropyl alcohol and, after centrifugation, the pellets were washed with $75 \%$ ethanol and used as total RNA.

\section{RT-PCR}

The pig MHC I $\alpha$ and $\beta 2 \mathrm{~m}$ genes were amplified ( $\alpha$-F: 5'-CCCGAATTCATGCGGG TCAGGGGCCCTCAAG-3', $\alpha$-R: 5 '-GCGTCGACCACTCTAGGATCCTTGGTAAG-3'; ß2m-F: 5'-CCCGAATTCTGATGGGGAAGGCGGCGGCGGT-3', $\beta 2 \mathrm{~m}-\mathrm{R}$ : 5'-GCGTCGAC TGGAACTCGGGATCCCACTTGT-3') with a one-step RT-PCR kit (Invitrogen). The PCR products were analyzed by $1.5 \%$ agarose gel electrophoresis and stained with ethidium bromide.

\section{Construction of Ii, Ii mutant, and MHC class I subunit expression vectors}

The cDNA fragments encoding pig MHC class I $\alpha$ and $\beta 2 \mathrm{~m}$ were subcloned into the expression vectors pDsRed2-N1 and pEGFP-N1 (both Clontech, USA). The cDNA fragment encoding pig Ii was made in our laboratory and subcloned into pEGFP-C1 (Clontech). Pig Ii mutant with a deleted CLIP region ( $\triangle$ CLIP-Ii) was constructed by overlap extension PCR. The first round of PCR was performed with primers Ii-F1: 5'-GGAAGATCTATGGAGGACCAG CGCGACCTC-3' (BglII) and RM: 5'-CGCATAGGCTCCGGGCCTTCCTTCATCCGCAGG CTCTCCA-3', and the full-length pig Ii was used as template. The second round of PCR was performed with primers FM: 5'-TGGAGAGCCTGCGGATGAAGGAAGGCCCGGAGCCT ATGCG-3' and Ii-R1: 5'-CCCGAATTCCAGGATGACTTGGCCGAGATCC-3' (EcoRI), and the full-length pig Ii was used as template. The underlined sequences are restriction enzyme sites. The third round PCR used the products from the first- and second-round PCR as template and used Ii-F1 and Ii-R1 as primers. PCR conditions were as follows: denature for $2 \mathrm{~min}$ at $95^{\circ} \mathrm{C}$, denature for $1 \mathrm{~min}$ at $94^{\circ} \mathrm{C}$, annealing for $1 \mathrm{~min}$ at $62^{\circ} \mathrm{C}$, and extension for $2 \mathrm{~min}$ at $972^{\circ} \mathrm{C}$. The third PCR products were cloned into pEGFP-C1. All PCR products were cloned into the appropriate eukaryotic expression vectors and sequence-verified.

\section{Preparation of the pig Ii fusion protein}

The full-length pig Ii was cloned into the pGEX-4T-1 (Amersham Bioscience, UK) to generate the recombinant prokaryotic expression vector pGEX-4T-1-Ii. The recombinant plasmid was transformed into Escherichia coli strain BL21 (Novagen) and protein expression was induced by addition of $1 \mathrm{mM}$ isopropyl- $\beta$-D-thiogalactopyranoside for $3.5 \mathrm{~h}$ at $37^{\circ} \mathrm{C}$. The cells were resuspended in buffer containing $50 \mathrm{mM}$ Tris-HCl, $\mathrm{pH}$ 8.0, $1 \mathrm{mM}$ EDTA, $2 \mathrm{mM}$ DTT, and $0.1 \mathrm{mM}$ PMSF and sonicated for 10 short pulses of $30 \mathrm{~s}$ each. After washing 3 times in inclusion bodies washing buffer ( $3 \%$ sucrose, $100 \mathrm{mM} \mathrm{NaCl}, 50 \mathrm{mM}$ Tris, $0.5 \%$ Triton $\mathrm{X}-100$ ), the pellets were suspended in buffer containing $10 \mathrm{mM}$ Tris, $\mathrm{pH} 8.0,150 \mathrm{mM} \mathrm{NaCl}, 1$ mM EDTA, and $2 \mathrm{mM}$ DTT with $1.5 \%$ sodium lauryl sarcosine. The supernatant was dialyzed against phosphate-buffered saline containing 3\% Triton X-100, then the fusion proteins were 
purified with glutathione-Sepharose 4B beads (Amersham Bioscience) according to manufacturer protocols and assayed by SDS-PAGE.

\section{Preparation of mouse polyclonal antibodies against pig Ii}

Eight mice were immunized intraperitoneally with $0.1 \mathrm{mg}$ fusion protein emulsified in an equal volume of complete Freund's adjuvant, then boosted 5 times with an emulsion of fusion protein and incomplete Freund's adjuvant at 2-week intervals. Two weeks after the last booster, the whole volume of immune serum was isolated. After titration, it was used for immunoprecipitation. Serum from non-immune mice was used as control.

\section{Cell culture and transient transfection of COS-7 cells}

COS-7 cell lines were maintained in RPMI 1640 medium supplemented with $10 \%$ fetal bovine serum (Gibco, USA). Briefly, 70\% confluent COS-7 cells were split into 6 wells (for immunoprecipitation) or 24 wells (with coverslips for immunofluorescence microscopy) 1 day before transfection. COS-7 cells were transfected with Lipofectamine 2000 (Invitrogen) according to manufacturer protocols.

\section{Fluorescence microscopy}

GFP or RFP expression marked positively transfected cells. At $36 \mathrm{~h}$ after transfection, cells on coverslips were fixed with $4 \%$ paraformaldehyde and visualized with an Olympus fluorescence microscope (Olympus, Tokyo, Japan).

\section{Immunoprecipitation and Western blotting}

The cells were lysed as previously described (Shachar et al., 1995) and pre-cleared with protein $G$ agarose (Amersham Pharmacia, Sweden). Immunoprecipitation was performed with mouse polyclonal antibodies against pig Ii and protein G sepharose. Washed immunoprecipitates were resolved by SDS-PAGE and blotted onto a PVDF membrane (Millipore, Germany). The blots were blocked with $10 \%(\mathrm{v} / \mathrm{v})$ fetal cattle serum for $1 \mathrm{~h}$ and then probed for $1 \mathrm{~h}$ with a mouse monoclonal anti-GFP (Clontech), followed by washing and 1-h incubation with horseradish peroxidase-conjugated goat anti-mouse IgG (Pierce, Rockford, USA) and peroxidase visualization by enhanced chemiluminescence (Amersham).

\section{RESULTS}

In order to measure the intracellular interaction of pig Ii (the CLIP domain) with MHC class I polypeptides, we amplified the pig MHC class I $\alpha$ and $\beta 2 \mathrm{~m}$ genes and constructed the following recombinant eukaryotic expression vectors: $p$ DsRed2-N1- $\alpha$, pEGFP-N1- $\alpha$, pDsRed2N1- $\beta 2 \mathrm{~m}$, and pEGFP-N1- $\beta 2 \mathrm{~m}$. MHC class I $\alpha$ and $\beta 2 \mathrm{~m}$ chains were 1095 and 357 bp in length, and had an open reading frame for a putative 365 or 119 amino acids. pEGFP-C1-Ii contains a gene segment of Ii. The pEGFP-C1- $\triangle$ CLIP-Ii contains Ii mutants with a deleted CLIP region. The pGEX-4T-1-Ii contains a gene segment of Ii. All recombinant constructs were sequence-verified. 


\section{Expression and localization of pig MHC class I subunits and Ii in COS-7 cells}

Control cells transfected with pDsRed2-N1, pEGFP-C1, and pEGFP-N1 showed strong cytoplasmic staining (Figure 1a, c, and f), while transfection with pEGFP-C1-Ii produced fluorescence in endomembrane vesicles (Figure 1b). Similarly, pEGFP-N1- $\alpha$, pEGFPN1- $\beta 2 \mathrm{~m}$, pDsRed2-N1- $\alpha$, and pDsRed2-N1- $\beta 2 \mathrm{~m}$ transfection produced fluorescence in endomembrane vesicles (Figure 1d, e, g, and h). Thus, fusions to the N-terminus of GFP or RFP ( $\alpha$-GFP, $\beta 2 \mathrm{~m}$-GFP, $\alpha$-RFP, and $\beta 2 \mathrm{~m}$-RFP) or to the C-terminus of GFP (GFP-Ii) retain the fluorescence properties of the native protein, allowing the fusion protein to be localized in vivo.

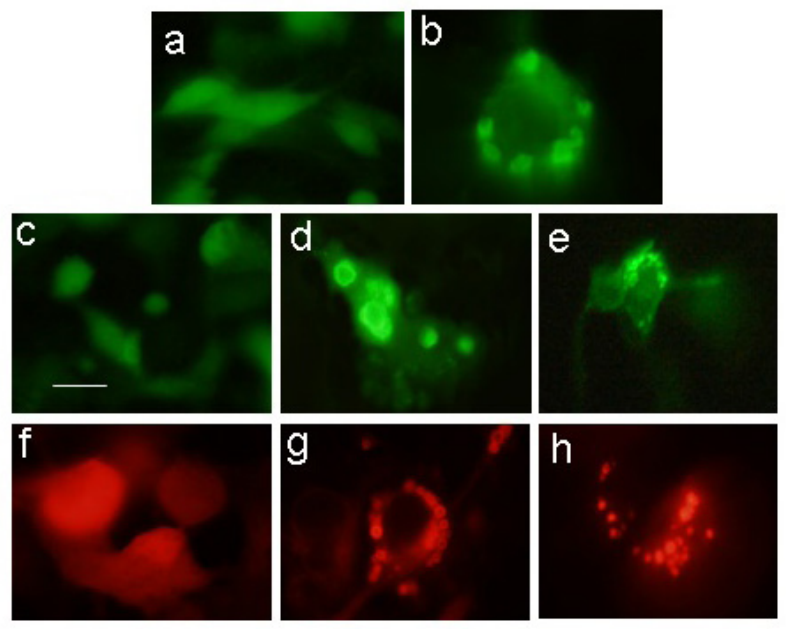

Figure 1. Expression and localization of pig major histocompatibility complex class I $\alpha, \beta 2 \mathrm{~m}$ and Ii in COS-7 cells. COS-7 cells transiently transfected with pEGFP-C1 (a), pEGFP-C1-Ii (b), pEGFP-N1 (c), pEGFP-N1- $\alpha$ (d), pEGFP-N1- $\beta 2 \mathrm{~m}$ (e), pDsRed2-N1 (f), pDsRed2-N1- $\alpha$ (g), and pDsRed2-N1- $\beta 2 \mathrm{~m}$ (h) were visualized by fluorescence microscopy. Bar $=10 \mu \mathrm{m}$.

\section{Colocalization of pig Ii and MHC I in COS-7 cells}

To evaluate the colocalization of Ii and mutant Ii with MHC class I $\alpha, \beta 2 \mathrm{~m}$ polypeptides, pEGFP-C1-Ii, pEGFP-C1- $\Delta$ CLIP-Ii, and pDsRed2-N1- $\alpha$ or pDsRed2-N1- $\beta 2 \mathrm{~m}$ were transiently cotransfected into COS-7 cells (Figure 2). Transfection with pDsRed2-N1- $\alpha$ or/and pDsRed2-N1- $\beta 2 \mathrm{~m}$ produced red fluorescence in endomembrane vesicles (Figure 2a, c, e, and $\mathrm{f}$ ), while pEGFP-C1-Ii and pEGFP-C1- $\triangle$ CLIP-Ii transfection produced green fluorescence (Figure 2b, d, and h). Three polypeptides (pEGFP-C1-Ii, pDsRed2-N1- $\alpha$ and pDsRed2-N1$\beta 2 \mathrm{~m}$ ) maintained intracellular localization, with yellow fluorescence showing Ii and MHC class I colocalization only in a portion of the green vesicles (Figure 2f), indicating oligomerization of the GFP-Ii and $\alpha$-RFP and $\beta 2 \mathrm{~m}$-RFP gene products. However, the unique yellow fluorescence was not observed in cells cotransfected pEGFP-C1- $\Delta$ CLIP-Ii and pDsRed2-N1- $\alpha$ and pDsRed2-N1- $\beta 2 \mathrm{~m}$ (Figure $2 \mathrm{~h}$ ). Normally, these genes are expressed in different parts of the cell, with no fluorescence overlap, but co-expression forms oligomers in the endomembranes to produce yellow fluorescence. 

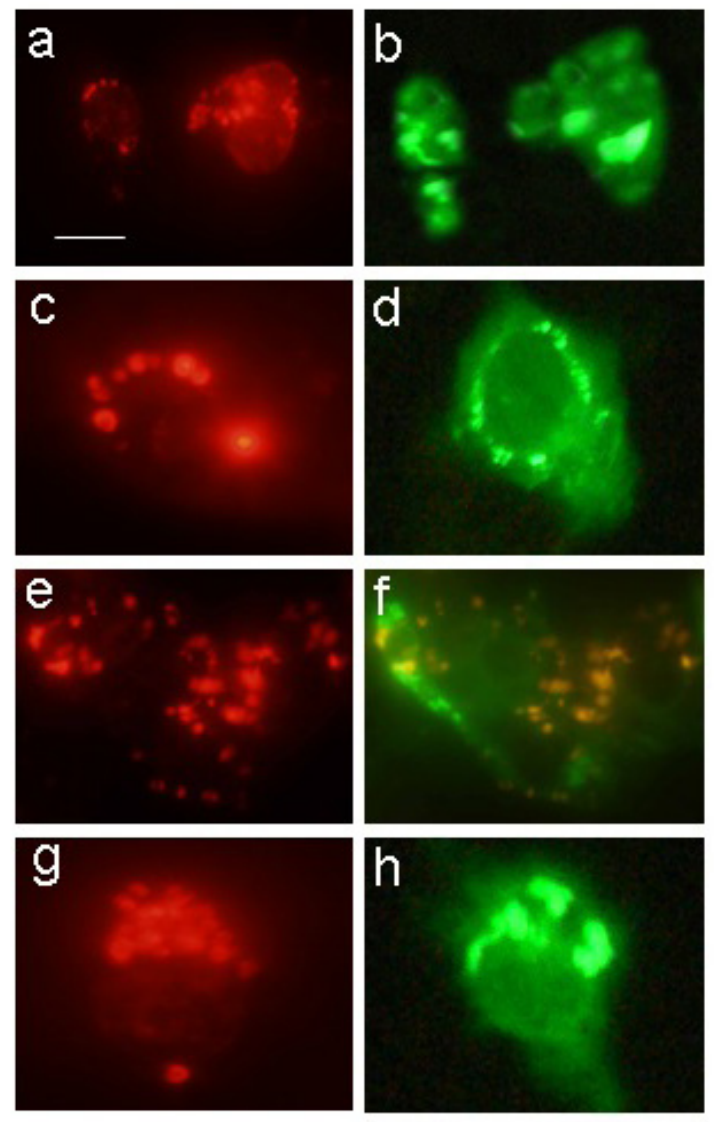

Figure 2. Colocalization of pig Ii and major histocompatibility complex class I (MHC I) $\alpha$ or $\beta 2 \mathrm{~m}$ chains in COS-7 cells. COS-7 cells transiently transfected with pEGFP-C1-Ii and pDsRed2-N1- $\alpha$ were visualized by fluorescence microscopy: a. $\alpha$-RFP, b. GFP-Ii in the same cell; cells transiently transfected with pEGFP-C1-Ii and pDsRed2N1- $\beta 2 \mathrm{~m}$ were visualized by fluorescence microscopy: c. $\beta 2 \mathrm{~m}$-RFP, $d$. GFP-Ii in the same cell; cells transiently transfected with pEGFP-C1-Ii, pDsRed2-N1- $\alpha$ and pDsRed2-N1- $\beta 2 \mathrm{~m}$ were visualized by fluorescence microscopy: e. $\alpha$-RFP and $\beta 2 \mathrm{~m}$-RFP, f. GFP-Ii in the same cell yellow indicating colocalization of GFP-Ii and $\alpha$-RFP and $\beta 2 \mathrm{~m}-\mathrm{RFP}$; cells transiently transfected with pEGFP-C1- $\Delta$ CLIP-Ii, pDsRed2-N1- $\alpha$ and pDsRed2-N1- $\beta 2 \mathrm{~m}$ were visualized by fluorescence microscopy: g. $\beta 2 \mathrm{~m}-\mathrm{RFP}$, h. GFP- $\triangle$ CLIP-Ii in the same cell. Bar $=10 \mu \mathrm{m}$.

\section{The Ii-MHC class I interaction}

The Ii-MHC class I interaction in COS-7 cells is shown in Figure 3. We performed immunoprecipitation with mouse antibodies against pig Ii, separation with SDS-PAGE, and immunoblotting for MHC class I using mAbs against GFP. When pEGFP-C1-Ii was cotransfected with pEGFP-N1- $\alpha$ or pDsRed2-N1- $\beta 2 \mathrm{~m}$, immunoprecipitation did not yield $\alpha$-GFP or $\beta 2 \mathrm{~m}$-GFP expression. Only when 3 constructs were cotransfected did oligomerization of GFPIi and $\alpha$-GFP and $\beta 2 \mathrm{~m}$-GFP occur. 


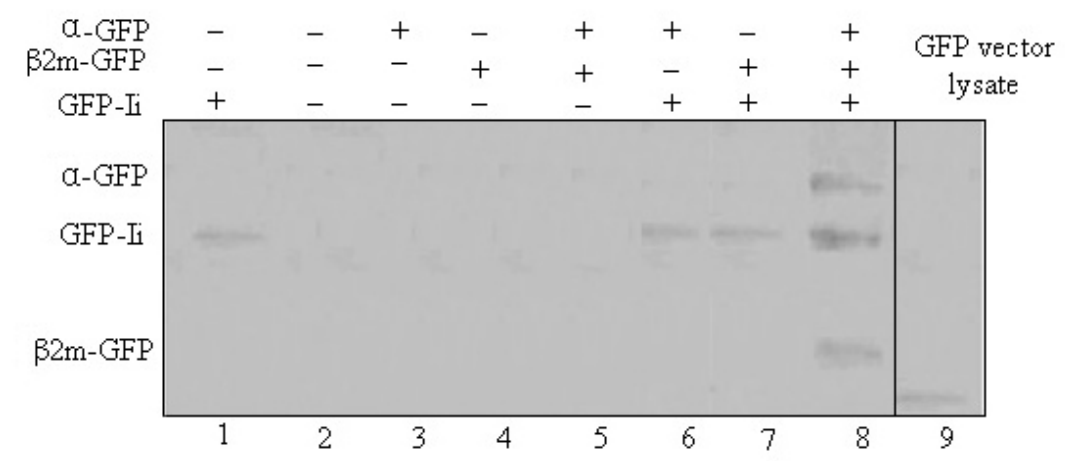

Figure 3. Interaction of pig Ii with major histocompatibility complex class I (MHC I). COS-7 cells were transfected with pEGFP-C1-Ii (lane 1), with pEGFP-N1- $\alpha$ (lane 3), with pEGFP-N1- $\beta 2 \mathrm{~m}$ (lane 4), with pEGFP-N1- $\alpha$ and pEGFP-N1- $\beta 2 \mathrm{~m}$ (lane 5), with pEGFP-C1-Ii and pEGFP-N1- $\alpha$ (lane 6 ) or pEGFP-N1- $\beta 2 \mathrm{~m}$ (lane 7), with pEGFPC1-Ii, pEGFP-N1- $\alpha$ and pEGFP-N1- $\beta 2 \mathrm{~m}$ (lane 8). Lane $2=$ COS-7 cell lysate; lane $9=$ COS-7 cells transfected with pEGFP-C1 empty vector lysate. Pig Ii was immunoprecipitated with mouse polyclonal antibodies against pig Ii; subsequently, MHC I $\alpha$ or/and $\beta 2 \mathrm{~m}$ polypeptides tagged with GFP were conducted with mAbs against GFP for Western blotting.

\section{CLIP fragment stabilization of MHC class I}

To determine whether Ii-MHC I interaction requires the CLIP binding segment in Ii; we tested the interaction of pEGFP-C1- $\triangle$ CLIP-Ii with pEGFP-N1- $\alpha$ or pEGFP-N1- $\beta 2 \mathrm{~m}$. Whether 2 (pEGFP-C1- $\Delta$ CLIP-Ii and pEGFP-N1- $\alpha$ or pEGFP-C1- $\Delta$ CLIP-Ii and pEGFP-N1$\beta 2 \mathrm{~m}$ ) or 3 constructs (pEGFP-C1- $\Delta$ CLIP-Ii, pEGFP-N1- $\alpha$ and pEGFP-C1- $\Delta$ CLIP-Ii) were cotransfected, immunoprecipitation was not associated with $\alpha$-GFP or $\beta 2 \mathrm{~m}$-GFP expression. Thus, Ii CLIP deletion blocked association with MHC I $\alpha$ and $\beta 2 \mathrm{~m}$ (Figure 4).

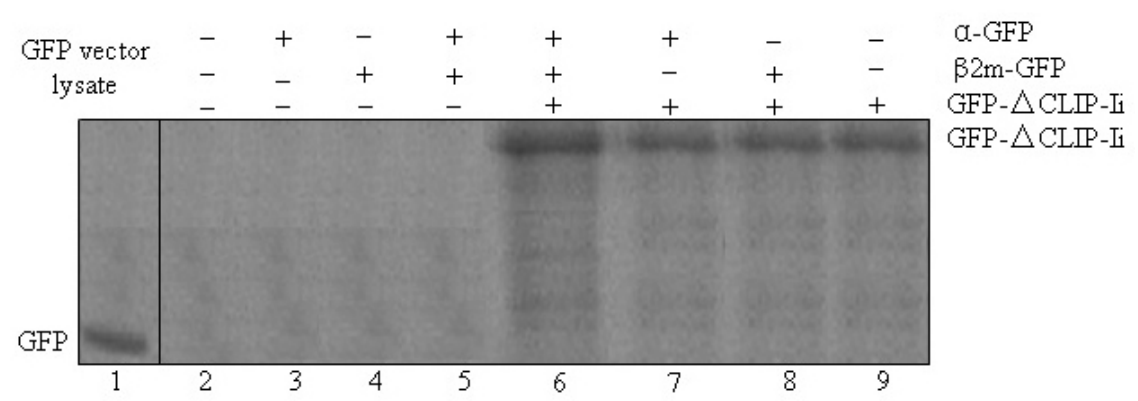

Figure 4. Interaction of class II-associated invariant chain peptide of Ii with major histocompatibility complex class I (MHC I). COS-7 cells were transfected with pEGFP-N1- $\alpha$ (lane 3), with pEGFP-N1- $\beta 2 \mathrm{~m}$ (lane 4), with pEGFP-N1- $\alpha$ and pEGFP-N1- $\beta 2 \mathrm{~m}$ (lane 5), with pEGFP-C1- $\Delta$ CLIP-Ii, pEGFP-N1- $\alpha$ and pEGFP-N1- $\beta 2 \mathrm{~m}$ (lane 6 ), with pEGFPC1- $\Delta$ CLIP-Ii and pEGFP-N1- $\alpha$ (lane 7), with pEGFP-C1- $\triangle$ CLIP-Ii and pEGFP-N1- $\beta 2 \mathrm{~m}$ (lane 8), with pEGFP-C1$\Delta$ CLIP-Ii. Lane $2=$ COS-7 cell lysate; lane $1=$ COS-7 cells transfected with pEGFP-C1 empty vector lysate. Pig pEGFP-C1- $\triangle$ CLIP-Ii was immunoprecipitated with mouse polyclonal antibodies against pig Ii; subsequently, MHC I $\alpha$ or $\beta 2 \mathrm{~m}$ polypeptides tagged with GFP were conducted with mAbs against GFP for Western blotting. 


\section{DISCUSSION}

GFP or RFP, as markers for gene expression, can monitor target gene expression and protein localization in live cells. In order to ensure correct expression of the fusion protein, we used a Signal P4.0 Server (http://www.cbs.dtu.dk/services/SignalP/) to analyze the N-terminal segment of MHC I $\alpha$ or $\beta$ chains containing the signal peptide, while the N-terminus of Ii contains a signal anchor. Signaling peptides and anchoring activity mediate arrival at the ER and maintenance of intracellular localization (Zhai et al., 2001). Therefore, we connected MHC I $\alpha$ and $\beta 2 \mathrm{~m}$ to the $\mathrm{N}$ terminus of GFP, RFP, or Ii to the C-terminus of GFP. This strategy allowed direct observation of the biological activity of the fusion protein.

It has been demonstrated that Ii is also capable of strong association with MHC class I molecules (Cerundolo et al., 1992; Vigna et al., 1996). This association appears to depend on prior binding of MHC class I with $\beta 2$-microglobulin and Ii does not associate with free class I heavy chain (Vigna et al., 1996). Fluorescence experiments confirmed that pig Ii cannot localize with single MHC molecular subunits; colocalization was observed only when 3 kinds of polypeptides ( $\alpha$-RFP, $\beta 2 \mathrm{~m}$-RFP and GFP-Ii) were cotransferred into COS-7 cells. Furthermore, immunoprecipitation indicated that MHC I $\alpha$ or MHC I $\beta 2 \mathrm{~m}$ were not effective in combination with Ii; Ii chain requires complete MHC I molecules to form the complex. Lin et al. (2009) reported that Ii, unlike other proteins that bind to MHC class I in the ER, does not bind to the open form of class I, although it can bind to the folded form. Sugita and Brenner (1994) showed that, in the presence of Ii, the secretory pathway of MHC class I was changed. Several reports have shown that exogenous antigens can be presented by MHC class I molecules to T cells in vivo and in vitro (Stone et al., 1978; Stam et al., 1990).

At present, the interaction mechanism of Ii and MHC class I molecules cannot be determined, but Ii and MHC class I can form complexes and reach the endosome (Neumann and Koch, 2006). MHC I molecules might be involved in exogenous antigen peptide antigen presentation. In addition, exogenous antigen peptide binds with MHC class I on the antigenpresenting cell surface, form the phagosome, and then MHC class I returns to the cell membrane recycling process. Ii chain may also act as directional guidance, guiding newborn MHC I molecules into the endosome. The interactions of Ii and MHC class I may in part explain the phenomenon of antigen cross-presentation.

The CLIP fragment of Ii binds to the MHC II $\alpha \beta$ dimer DR3 (Ghosh et al., 1995) and the MHC II groove contains several anchor positions for CLIP binding (Liang et al., 1996; Serwe et al., 1997). Powis (2006) performed site-directed mutagenesis of Ii cDNA to alter the methionine at positions 91 and 99 to alanine. A pulse-chase experiment was also performed using a 15-min label and up to 90-min chase followed by immunoprecipitation; the results demonstrated that the Ii-MHC class I interaction was lost between the 45- and 90-min chase. In our experiment, a pig Ii mutant with a deleted CLIP cannot bind MHC I $\alpha$ and $\beta$ chain. The ability of Ii to interact with MHC class I through binding of CLIP to the peptide groove suggests that it may incorporate into the MHC class I peptide-loading complex.

\section{ACKNOWLEDGMENTS}

Research supported by a grant from the National Natural Science Foundation of China (\#31101797) and the President of Anhui Agriculture University Youth Fund Project (\#2010zd06). 


\section{REFERENCES}

Basha G, Omilusik K, Chavez-Steenbock A, Reinicke AT, et al. (2012). A CD74-dependent MHC class I endolysosomal cross-presentation pathway. Nat. Immunol. 13: 237-245.

Cerundolo V, Elliott T, Elvin J, Bastin J, et al. (1992). Association of the human invariant chain with H-2 Db class I molecules. Eur. J. Immunol. 22: 2243-2248.

Cresswell P (1992). Chemistry and functional role of the invariant chain. Curr. Opin. Immunol. 4: 87-92.

Donaldson JG and Williams DB (2009). Intracellular assembly and trafficking of MHC class I molecules. Traffic 10: 1745-1752.

Ghosh P, Amaya M, Mellins E and Wiley DC (1995). The structure of an intermediate in class II MHC maturation: CLIP bound to HLA-DR3. Nature 378: 457-462.

Liang MN, Lee C, Xia Y and McConnell HM (1996). Molecular modeling and design of invariant chain peptides with altered dissociation kinetics from class II MHC. Biochemistry 35: 14734-14742.

Lin X, Wang X, Capek HL, Simone LC, et al. (2009). Effect of invariant chain on major histocompatibility complex class I molecule expression and stability on human breast tumor cell lines. Cancer Immunol. Immunother. 58: 729-736.

Neumann J and Koch N (2006). A novel domain on HLA-DRbeta chain regulates the chaperone role of the invariant chain. J. Cell Sci. 119: 4207-4214.

Odorizzi CG, Trowbridge IS, Xue L, Hopkins CR, et al. (1994). Sorting signals in the MHC class II invariant chain cytoplasmic tail and transmembrane region determine trafficking to an endocytic processing compartment. J. Cell Biol. 126: 317-330.

Peters PJ, Neefjes JJ, Oorschot V, Ploegh HL, et al. (1991). Segregation of MHC class II molecules from MHC class I molecules in the Golgi complex for transport to lysosomal compartments. Nature 349: 669-676.

Powis SJ (2006). CLIP-region mediated interaction of Invariant chain with MHC class I molecules. FEBS Lett. 580: 3112-3116.

Reber AJ, Turnquist HR, Thomas HJ, Lutz CT, et al. (2002). Expression of invariant chain can cause an allele-dependent increase in the surface expression of MHC class I molecules. Immunogenetics 54: 74-81.

Roche PA and Cresswell P (1990). Invariant chain association with HLA-DR molecules inhibits immunogenic peptide binding. Nature 345: 615-618.

Romagnoli P and Germain RN (1994). The CLIP region of invariant chain plays a critical role in regulating major histocompatibility complex class II folding, transport, and peptide occupancy. J. Exp. Med. 180: 1107-1113.

Serwe M, Reuter G, Sponaas A, Koch S, et al. (1997). Both invariant chain isoforms Ii31 and Ii41 promote class II antigen presentation. Int. Immunol. 9: 983-991.

Shachar I, Elliott EA, Chasnoff B, Grewal IS, et al. (1995). Reconstitution of invariant chain function in transgenic mice in vivo by individual $\mathrm{p} 31$ and $\mathrm{p} 41$ isoforms. Immunity $3:$ 373-383.

Stam NJ, Vroom TM, Peters PJ, Pastoors EB, et al. (1990). HLA-A- and HLA-B-specific monoclonal antibodies reactive with free heavy chains in western blots, in formalin-fixed, paraffin-embedded tissue sections and in cryo-immunoelectron microscopy. Int. Immunol. 2: 113-125.

Stone KR, Mickey DD, Wunderli H, Mickey GH, et al. (1978). Isolation of a human prostate carcinoma cell line (DU 145). Int. J. Cancer 21: 274-281.

Sugita M and Brenner MB (1994). An unstable beta 2-microglobulin: major histocompatibility complex class I heavy chain intermediate dissociates from calnexin and then is stabilized by binding peptide. J. Exp. Med. 180: 2163-2171.

Sugita M and Brenner MB (1995). Association of the invariant chain with major histocompatibility complex class I molecules directs trafficking to endocytic compartments. J. Biol. Chem. 270: 1443-1448.

Teyton L, O'Sullivan D, Dickson PW, Lotteau V, et al. (1990). Invariant chain distinguishes between the exogenous and endogenous antigen presentation pathways. Nature 348: 39-44.

Tulp A, Verwoerd D, Dobberstein B, Ploegh HL, et al. (1994). Isolation and characterization of the intracellular MHC class II compartment. Nature 369: 120-126.

Van Bleek GM and Nathenson SG (1990). Isolation of an endogenously processed immunodominant viral peptide from the class I H-2Kb molecule. Nature 348: 213-216.

Vigna JL, Smith KD and Lutz CT (1996). Invariant chain association with MHC class I: preference for HLA class I/ $\beta$ 2-microglobulin heterodimers, specificity, and influence of the MHC peptide-binding groove. J. Immunol. 157: 45034510.

Zhai ZH, Wang XZ and Ding MX (2001). Cell Biology. Volume 192. Higher Education Press, Beijing, 164-202.

Zhong D, Yu W, Liu Y, Liu J, et al. (2004). Molecular cloning and expression of two chicken invariant chain isoforms produced by alternative splicing. Immunogenetics 56: 650-656. 